\title{
Der zeitliche Anstieg des Stromes in die anomale Glimmentladung
}

(Einfluß der Raumladung auf Gasverstärkung und Nachlieferung bei Drucken unter 1 Torr für $\mathrm{H}_{2}$ und $\mathrm{O}_{2}$ ) Hans SсHLumbohm

Institut für Angewandte Physik der Universität Hamburg

(Z. Naturforschg. 22 a, 1255-1260 [1967] ; eingegangen am l. April 1967)

\begin{abstract}
Measurements of the current rise of a low pressure discharge in $\mathrm{H}_{2}$ and $\mathrm{O}_{2}$ at $p d<5 \mathrm{Torr} \cdot \mathrm{cm}$ under homogenous field conditions have shown that a direct transition from the Townsend- into the anomalous glow-discharge takes place without any change of the voltage across the gap. The current growth is explained by the space-charge-induced variations of the electric field on the gas amplification $e^{a d}$ and on the secondary ionisation coefficient $\gamma$. At small current densities the space charge causes an increase of the coefficient $\mu=\gamma\left(e^{a d}-1\right)$ by its influence on $\gamma$ whereas at higher current densities this initial increase is reduced by a decrease of $e^{\alpha d}$. The current growth rate is calculated using an approximate equation connecting the relative rise $\mathrm{d} \ln J / \mathrm{d} t$ and $\mu$ and is in agreement with the experimental values.

At a certain current density the space charge influence on the secondary ionisation and on the gas amplification balance each other and the steady-state condition $\mu=1$ is again fulfilled. The current growth thus ends in a steady anomalous glow discharge state which is determined by both the voltage across the gap and the current density corresponding to $\mu=1$.
\end{abstract}

Wird an der Kathode einer Parallelplatten-Entladungsstrecke eine hohe Zahl von Elektronen gleichzeitig ausgelöst, setzt bei Anlegen eines elektrischen Feldes ein Stromanstieg ein, der bei hohem Druck ( $>$ einige 10 Torr) bis zum stromstarken Funken führt und bei niedrigen Drucken (unterhalb einiger Torr) in einer stationären Glimmentladung endet.

Ziel der vorliegenden Arbeit ist die Untersuchung der Stromanstiege bei niedrigen Drucken. Im Gegensatz zu hohen Drucken (siehe die Zusammenstellung in ${ }^{1}$ ) verursacht die durch Raumladung bedingte Feldverzerrung für niedrige Drucke eine Verringerung der Stoßionisation ${ }^{2}$. Da aber dennoch der Strom vom Lawinen- in das Glimmstadium um das etwa $10^{4}$-fache ansteigt, vermutet man eine stromfördernde Wirkung der Raumladung durch Beeinflussung der Nachlieferung.

Ein weiteres Ziel war es zu klären, ob der bei niedrigen Drucken beobachtete Utbergang der Stromanstiege in die Glimmentladung statt in einen stromstarken Funken sich als Folge der unterschiedlichen Wirkung der Feldverzerrung verstehen läßt.

\section{Die verwendete Meßmethodik}

Die verwendete Parallelplatten-Entladungsstrecke bestand aus Messingelektroden mit einer ebenen Fläche

1 H. RaEther, Electron Avalanches and Breakdown in Gases, Butterworths, London 1964. von $300 \mathrm{~cm}^{2}$ und einem Randbereich von $60 \mathrm{~cm}^{2}$. Durch die als feines Netz ausgebildete Anode trat das Licht eines UV-Blitzes auf die Kathode und löste über die Fläche verteilt wahlweise $10^{4}$ bis $10^{6}$ Elektronen innerhalb etwa 15 nsec aus. Gemessen wurde der hierdurch verursachte Stromanstieg, der sich unter dem Einfluß eines konstant anliegenden elektrischen Feldes entwickelt. Der Entladungskreis war so ausgelegt, daß die zwischen den Elektroden liegende Gleichspannung während der jeweiligen Beobachtungszeit der Stromanstiege nicht absank. Die Apparatur und die Meßmethode wurden ausführlicher in ${ }^{\mathbf{3}}$ beschrieben.

\section{Experimentelle Ergebnisse}

Messungen des zeitlichen Stromanstiegs haben wir in Wasserstoff und Sauerstoff bei verschiedenen Drucken unterhalb 1 Torr durchgeführt. Dies geschah in folgender Weise: Die an der Strecke liegende Spannung wurde solange gesteigert, bis der durch einen UV-Blitz ausgelöste Strom gerade nicht mehr abbrach, sondern selbständig wurde. Dieser Übergang geschah in einem Spannungsintervall der relativen Breite einiger $10^{-4}$ und definiert damit sehr genau die Zündspannung. Bei Primärelektronendichten von $10^{2}$ bis $10^{3}$ pro $\mathrm{cm}^{2}$ beginnt der Strom mit Dichten von $10^{-9}$ bis $10^{-8} \mathrm{~A} / \mathrm{cm}^{2}$ (Lawinenstadium mit sehr schwacher Raumladung, $e^{a d} \sim 30, T_{+} \sim$ einige $\mu \mathrm{sec}$ ), steigt um das $10^{3}$ - bis

2 A. von Engel u. M. Steenbeck, Elektrische Gasentladungen, Band 2, Springer-Verlag, Berlin 1934.

3 H. Sснцимвонм, Z. Phys. 182, 306 [1965]. 
$5 \cdot 10^{4}$-fache und geht dann in ein stationäres Stadium mit Stromdichten von $10^{-5}$ bis $5 \cdot 10^{-4} \mathrm{~A} / \mathrm{cm}^{2}$ je nach gewähltem Druck über, ohne daß die an der Strecke liegende Spannung abgesunken ist. Der Widerstand, über dem der Strom gemessen wurde, war stets so klein gewählt, daß auch bei den höchsten Strömen der Spannungsabfall die an der Strecke liegende Spannung nicht beeinflußte. Die beobachtete Leuchterscheinung weist dieses Endstadium als anomale Glimmentladung aus.

Wir haben Oszillogramme über den gesamten Bereich vom Start der Primärelektronen bis zum Glimmstadium in aneinander anschließenden Empfindlichkeitsstufen photographiert. Abb. 1 zeigt einen Stromanstieg unmittelblar nach dem Start der Primärelektronen. Da die Zeitablenkung sich über viele Ionenlaufzeiten erstreckt, ist die Generationenstruktur des Stromes nicht aufgelöst. Abb. 2 zeigt den Übergang des inzwischen auf das $10^{3}$-fache angestie-

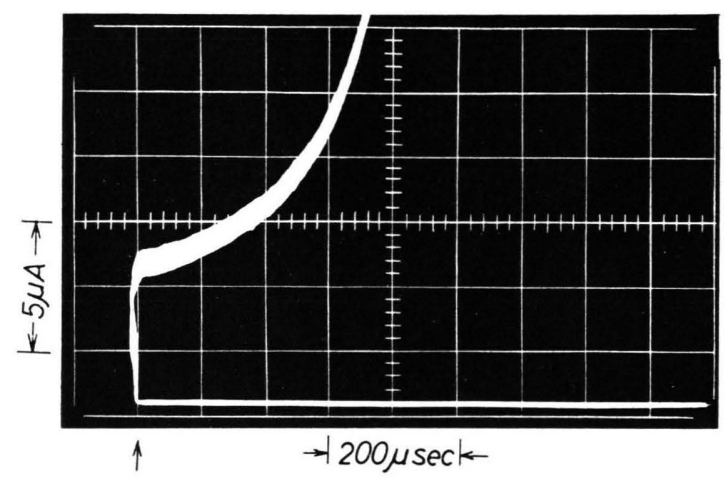

Abb. 1. Zeitlicher Anstieg des Stromes nach Auslösung der Primärelektronen. Der eingezeichnete Pfeil markiert die Zeit der Auslösung. Wasserstoff : $p=0,19$ Torr, $d=4,7 \mathrm{~cm}$, $E / p=290$ Volt $/ \mathrm{cm} \cdot$ Torr.

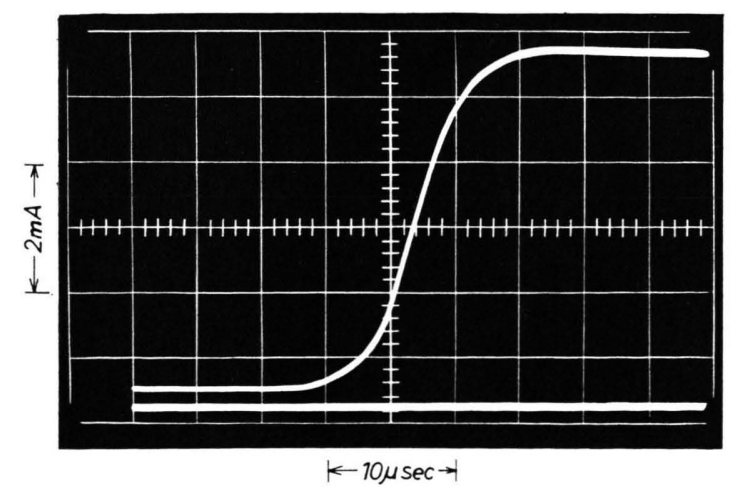

Abb. 2. Zeitlicher Verlauf des Stromes beim Übergang in das stationäre Endstadium ( 10 $0^{3}$-mal höherer Strom und 20-fach schnellere Zeitablenkung als in Abb. 1). Gleiche Bedingungen wie in Abb. 1. genen Stromes in den zeitlich konstanten Endwert. Um diesen Übergang zeitlich aufzulösen, ist dieses Oszillogramm mit 20-fach höherer Zeitablenkung geschrieben als das Oszillogramm der Abb. 1. Die Zeitachse erfaßt daher nur einen kleinen Ausschnitt aus der Gesamtentwicklung.

Sämtliche Anstiege haben wir halblogarithmisch umgezeichnet, wobei sich zeigte, daß die nacheinander unter gleichen Bedingungen gemessenen Ströme innerhalb einer geringen Streuung sich gleich verhielten. Daher ist es möglich, durch Aneinanderfügen der nacheinander bei den unterschiedlichen Stromhöhen ausgeführten Messungen quantitativ den gesamten Stromanstieg vom Start der Primärelektronen bis zum Glimmstadium zu erhalten.

Die Auswertung ergab stets bei geringen Stromstärken vom Start des Stromes an mit der verwendeten langsamen Zeitablenkung einen zeitlich überexponentiellen Anstieg (Abb. 1). Der Strom steigt überexponentiell etwa auf den $10^{3}$-fachen Anfangswert. Daran anschließend steigt der Strom nur noch exponentiell weiter an und strebt dann über einen unterexponentiellen Anstieg einem zeitlich konstanten Endwert zu. Abb. 2 zeigt diese Endphase des Anstiegs, zu dessen zeitlicher Auflösung eine 20-mal höhere Zeitablenkung gegenüber Abb. 1 benutzt wurde.

Zur quantitativen Diskussion haben wir die relative zeitliche Änderung

$$
\mathrm{d} \ln J / \mathrm{d} t=(\mathrm{d} J / \mathrm{d} t) / J
$$

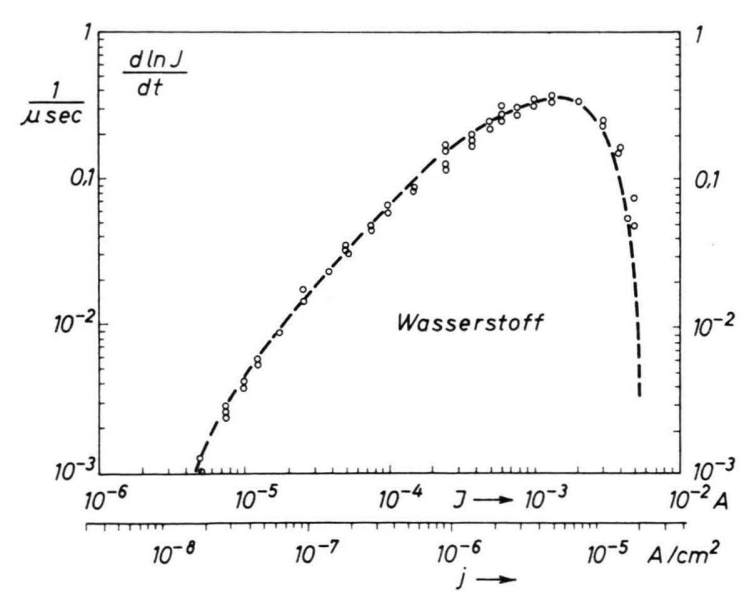

Abb. 3. Gemessene und berechnete Werte des Anstiegsverhaltens $\mathrm{d} \ln \mathrm{J} / \mathrm{d} t$ des Stromes in Abhängigkeit von Strom und Stromdichte. $\bigcirc \circ \circ \circ$ sind die experimentellen Werte, --der berechnete Verlauf. Wasserstoff : $p=0,19$ Torr, $d=4,7 \mathrm{~cm}$, $E / p=290 \mathrm{Volt} / \mathrm{cm} \cdot$ Torr $\left(\varepsilon_{0}=-2,3 \cdot 10^{-3}, \mathrm{~s}\right.$. Text $)$. 
des Stromes $J(t)$ gegen den Strom selbst (nicht gegen die Zeit) aufgetragen. Diese Größe erhält man unmittelbar durch halblogarithmische Umzeichnung der Stromanstiege $J(t)$. Die Gegenüberstellung des momentanen Anstiegs $\mathrm{d} \ln J / \mathrm{d} t$ gegen $J$ ist physikalisch sinnvoll, da die Stromentwicklung wesentlich durch die Raumladung, d. h. aber durch die Höhe des Stromes selbst bestimmt ist. Abb. 3 und 6 zeigen die für je eine Messung in Wasserstoff und Sauerstoff erhaltenen Auftragungen. - Diese Diagramme lassen sich leicht interpretieren: $\mathrm{Da}$ die Konstanz von $\mathrm{d} \ln J / \mathrm{d} t$ gegen $J$ einen zeitlich exponentiellen Stromanstieg bedeutet, besagt mit dem Strom zunehmendes $\mathrm{d} \ln J / \mathrm{d} t$ zeitlich überexponentiellen Anstieg und fallendes $\mathrm{d} \ln J / \mathrm{d} t$ unterexponentiellen Anstieg.

\section{Zur Deutung der experimentellen Ergebnisse}

Zur Deutung der gemessenen Stromentwicklung haben wir Stromanstiege berechnet und mit den experimentellen verglichen. Hierbei zeigte es sich, daß das Zusammenwirken von Gasverstärkung und Nachlieferung die Stromentwicklung erklärt, wenn die Einflüsse der Feldverzerrung auf Gasverstärkung und Nachlieferung entsprechend berücksichtigt werden.

Der nach dem Start des Stromes einsetzende zeitlich überexponentielle Anstieg entsteht durch die ständige Zunahme der Nachlieferung als Folge der Erhöhung der Feldstärke an der Kathode. Nach einem Stromanstieg auf etwa den 500-fachen Anfangswert hat die Feldverzerrung so stark zugenommen, daß neben der Zunahme der Nachlieferung eine Abnahme der Gasverstärkung merklich wird. Die Gasverstärkung nimmt mit weiter ansteigendem Strom schnell ab und kompensiert bei einem bestimmten Wert der Stromdichte die Erhöhung der Nachlieferung. Dieser Raumladungseinfluß auf die Gasverstärkung erklärt somit die Verlangsamung der Stromanstiege und den Übergang in einen stationären Endstrom.

Dieses Ergebnis haben wir durch näherungsweise Erfassung der Raumladungseinflüsse bei niedrigem Strom und numerische Rechnung bei höheren Strömen erhalten.

\section{a) Niedrige Stromdichte (Schwache Feldverzerrung)}

Die den Stromanstieg bestimmende Rückwirkung $\mu=\gamma\left(e^{\alpha d}-1\right)$ wird bei kleinen Variationen von $\gamma$ und $\alpha d$ eine Änderung

$$
\Delta \mu=\mu_{0} \cdot\left\{\Delta \gamma / \gamma_{0}+\Delta(\alpha d) /\left(1-e^{-\alpha_{0} d}\right)\right\}
$$

erfahren. In Abhängigkeit von der Raumladung, deren Höhe sich durch die Stromdichte ausdrücken läßt (siehe Anhang), erhält man für Wasserstoff bei $E / p=290 \mathrm{Volt} / \mathrm{cm} \cdot$ Torr, $d=4,7 \mathrm{~cm}, p=0,19$ Torr $\left(e^{\alpha_{0} d}=16, \gamma_{0} \cong 6,7 \cdot 10^{-2}\right)$.

$\Delta \mu \cong \mu_{0}\left\{5,58 \cdot 10^{8} \cdot j-1,03 \cdot 10^{11} \cdot j^{2}\right\} ; \quad j$ in $\mathrm{A} / \mathrm{cm}^{2}$.

Der erste Term der Klammer, der gleich $\Delta \gamma / \gamma_{0}$ ist, berechnet sich auf Grund der Annahme, daß die Höhe der Nachlieferung durch die Feldstärke an der Kathode bestimmt ist. Zur Rechnung ist der in ${ }^{4} \mathrm{ex}$ perimentell bestimmte $\gamma=\gamma(E / p)$-Zusammenhang benutzt und darin $E=E_{\text {Kathode }}$ gesetzt. Da unter den gewählten Bedingungen sicherlich die Elektronennachlieferung zum Teil durch positive Ionen erfolgt und keine Photonachlieferung auftritt ${ }^{4}$, scheint dies beim Stand der Kenntnisse die angemessenste Beschreibung zu sein. Mit zunehmender Stromdichte $j$ steigt die Feldstärke an der Kathode und als Folge hiervon auch $\gamma$, da die hier auftretenden $E / p$ in einem Bereich liegen, in dem die $\gamma(E / p)$-Kurve wachsendes $\gamma$ mit zunehmendem $E / p$ liefert.

Der zweite Term in der Klammer beschreibt die Änderung der Stoßionisation $\Delta(\alpha d)$ und wurde aus

$$
\frac{1}{2} \mathrm{~d}^{2} \alpha / \mathrm{d} E^{2} \int_{0}^{d}\left(E(x)-E_{0}\right)^{2} \mathrm{~d} x
$$

berechnet. Die bei unserem Experiment auftretenden Feldstärken liegen in dem Bereich, in dem $\mathrm{d}^{2} \alpha / \mathrm{d} E^{2}$ $<0$ ist (d. i. bei Wasserstoff bei $E / p$ oberhalb $70 \mathrm{~V}$ $/ \mathrm{cm} \cdot$ Torr). Die Gasverstärkung erfährt also eine Absenkung, denn das Integral liefert stets einen positiven Faktor zu d $\mathrm{d}^{2} \alpha / \mathrm{d} E^{2}$. Da nun der Term $\Delta(\alpha d)$ bis zu Stromdichten $j \sim 10^{-6} \mathrm{~A} / \mathrm{cm}^{2}$ bei sehr niedrigen Werten bleibt, erfährt $\mu$ eine Erhöhung $\Delta \mu$ $(\Delta \mu>0)$, die einzig durch die Zunahme der Nachlieferung bestimmt ist.

In Abb. 4 ist $\Delta \gamma / \gamma_{0} \cong \ln \left(\gamma / \gamma_{0}\right)$ [bei höheren Strömen $\left.\ln \left(\gamma / \gamma_{0}\right)\right]$ gegen den Strom aufgetragen. Es steigt $\Delta \gamma / \gamma_{0}$ mit wachsendem Strom über den ganzen Bereich an. Abb. 5 zeigt $\mu=\mu_{0}+\Delta \mu$ ebenfalls gegen den Strom aufgetragen. Der mit dem Strom ansteigende Teil der Kurve entsteht durch die $\mathrm{Zu}$ nahme der Nachlieferung. Entsprechend der an der Strecke liegenden Spannung ist $\mu=\mu_{0} \cong 1$ bei Strömen $J$ unter $10 \mu \mathrm{A}$ und steigt dann bis auf $1,3 \mathrm{mit}$ zunehmendem Strom an.

${ }^{4}$ Н. Sснцимвонм, Z. Naturforschg. 22 a, 347 [1967]. 


\section{b) Hohe Stromdichte (Starke Feldverzerrung)}

Da die Abnahme der Stoßionisation $\Delta(\alpha d)$ mit zunehmender Stromdichte schneller zunimmt als die Nachlieferung $\gamma$ [in der Näherungsdarstellung ist $\Delta(\alpha d)$ proportional dem Quadrat von $j$ und $\Delta \gamma / \gamma_{0}$ nur proportional $j$ ], gewinnt diese von einigen $100 \mu \mathrm{A}$ an ebenfalls Einfluß auf die Stromentwicklung. Da die Näherungsdarstellung in diesem Bereich nicht mehr anwendbar ist, haben wir die Änderung der Gasverstärkung mittels numerischer Rechnung bestimmt. In Abb. 4 ist der Logarithmus des Verhältnisses der Gasverstärkungen mit und ohne Raumladung

$$
\ln \left\{\left(\exp \left[\int_{0}^{d} \alpha \mathrm{d} x\right]-1\right) /\left(e^{a_{0} d}-1\right)\right\}
$$

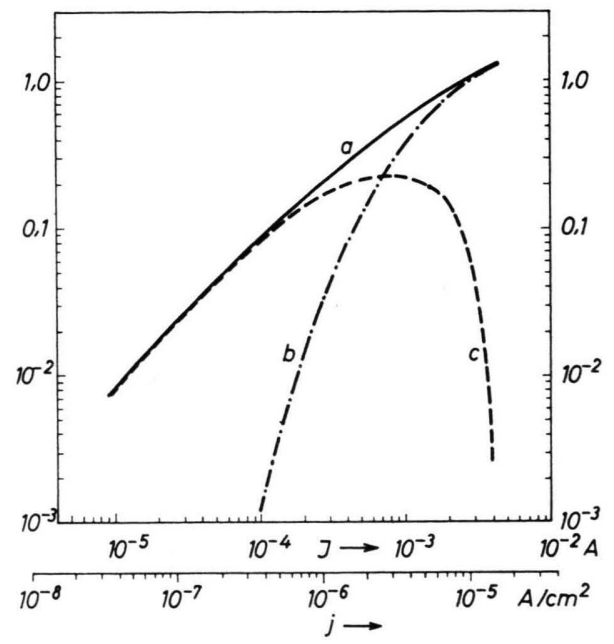

Abb. 4. Berechnete Werte der durch die Feldverzerrung veränderten Nachlieferung $\ln \left(\gamma / \gamma_{0}\right)$ [Kurve a], der Gasverstärkung $-\ln \left[\left(\exp \int_{0}^{t} \alpha \mathrm{d} x-1\right) /\left(e^{\alpha_{0} d}-1\right)\right] \quad$ [Kurve b] und der Rückwirkung $\ln \left(\stackrel{0}{\mu} / \mu_{0}\right)$ [Kurve c] in Abhängigkeit von Strom und Stromdichte. Gleiche Bedingungen wie Abb. 3 .

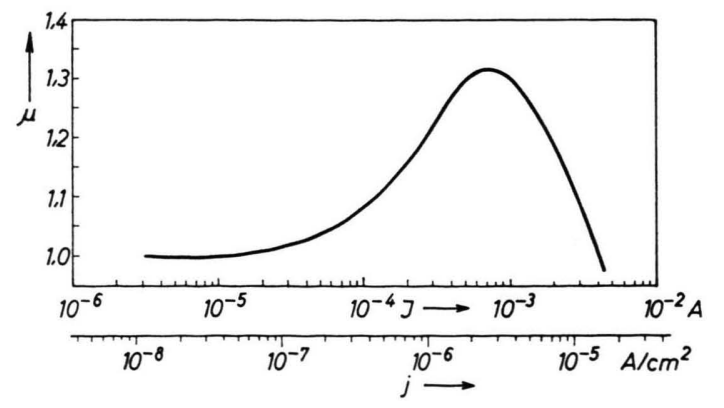

Abb. 5.

Berechnete Werte der Rückwirkung $\mu=\gamma\left(\exp \left[\int_{0}^{d} \alpha \mathrm{d} x\right]-1\right)$ in Abhängigkeit von Strom und Stromdichte. Gleiche Bedin. gungen wie Abb. 3. dem Betrage nach aufgetragen. Bei kleinen Werten ist dies gleich $|\Delta(\alpha d)|$. Man erkennt, da $\beta$ erst bei $J \approx 200 \mu \mathrm{A}|\Delta(\alpha d)|$ mit $10^{-2}$ die Höhe erreicht, die $\Delta \gamma / \gamma_{0}$ bereits bei $J=10 \mu \mathrm{A}$ hat. Es steigt $|\Delta(a d)|$ dann sogar schneller als quadratisch und erreicht bei $4 \mathrm{~mA}$ die Höhe von $\ln \left(\gamma / \gamma_{0}\right)$.

Das Zusammenwirken beider Einflüsse - die Zunahme der Nachlieferung und die Abnahme der Gasverstärkung - zeigt die gestrichelte Kurve in Abb. 4, die $\ln \left(\mu / \mu_{0}\right)$ angibt, das bei kleinem Strom gleich $\Delta \mu / \mu_{0}$ ist. Diese Kurve ergibt sich durch Addition der Einzeleinflüsse

$$
\ln \left(\mu / \mu_{0}\right)=\ln \left(\gamma / \gamma_{0}\right)+\ln \frac{\left(\exp \left[\int_{0}^{d} a \mathrm{~d} x\right]-1\right)}{\left(\exp \left[\alpha_{0} d\right]-1\right)} .
$$

Der erste Term der rechten Seite ist positiv, der zweite Term hat nur negative Werte. Solange der zweite Term klein ist, nimmt also $\ln \left(\mu / \mu_{0}\right)$ zu. Kommt aber der zweite Term in die Höhe des ersten, fällt $\ln \left(\mu / \mu_{0}\right)$ stark ab. Den Verlauf von $\mu$ zeigt Abb. 5. Abb. 4 entsprechend ist bei niedrigem Strom $\mu=\mu_{0} \cong 1$, steigt dann bis 1,3 an und fältt dann wegen der Abnahme der Gasverstärkung wieder auf $\mu=1 \mathrm{ab}$. Über diese Stromhöhe geht der Stromanstieg nicht hinaus, da für höheren Strom $\mu$ unterhalb 1 absinken würde.

Aus dem berechneten $\ln \left(\mu / \mu_{0}\right)$ läßt sich nun die momentane Stromänderung $d \ln J / d t$ angeben (siehe Anhang). In das Diagramm der Meßwerte Abb. 3 ist der Verlauf als gestrichelte Kurve eingetragen. Der berechnete Verlauf wurde hierzu durch günstigste Wahl zweier Parameter angepaßt: Die vom Strom bedeckte Fläche $F$ zur Umrechnung von Stromdichte $j$ auf den Strom $J$ wurde so gewählt, daß im Bereich der höheren Ströme möglichst gute UUbereinstimmung besteht. Es ergab sich dabei ein Wert von $F=270$ $\mathrm{cm}^{2}$; das ist mit $90 \%$ des ebenen Teiles der Elektroden gerade die Fläche, über die die Primärelektronen ausgelöst wurden. Der genaue Wert des durch die angelegte Spannung bestimmten $\mu=\mu_{0}$ beeinflußt die Höhe der berechneten Kurve bei niedrigen Strömen, wo die Raumladungswirkung noch schwach ist. Für günstigsten Angleich ergab sich ein Wert $\mu_{0}=1-2,3 \cdot 10^{-3} \quad\left(\varepsilon_{0}=\mu_{0}-1=-2,3 \cdot 10^{-3}\right)$ geringfügig unterhalb 1 . Das besagt, daß der Strom nur durch die fördernde Wirkung der Raumladung während der ersten Lawinengenerationen selbständig wird. 
Weitere unter anderen Entladungsbedingungen gemessene Stromanstiege haben wir ebenfalls mit berechneten verglichen. Hierbei ergab sich ebenso gute Übereinstimmung. Abb. 6 zeigt das Ergebnis einer Messung für Sauerstoff.

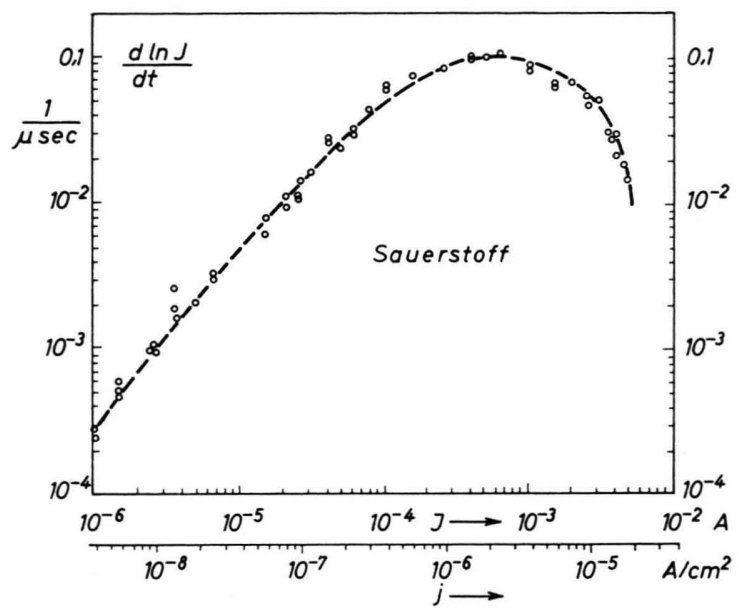

Abb. 6. Gemessene und berechnete Werte des Anstiegsverhaltens $\mathrm{d} \ln J / \mathrm{d} t$ des Stromes in Abhängigkeit von Strom und Stromdichte. $0 \circ \circ$ sind die experimentellen Werte, --- der berechnete Verlauf. Sauerstoff : $p=0,06$ Torr, $d=4,7 \mathrm{~cm}$, $E / p=1860 \mathrm{Volt} / \mathrm{cm} \cdot$ Torr $\left(\varepsilon_{0}=-1,2 \cdot 10^{-3}, \mathrm{~s}\right.$. Text $)$.

\section{Bemerkung zum Mechanismus der Glimm- entladung}

Wenn der Strom das stationäre Endstadium erreicht hat, zeigt die Entladungsstrecke eine Leuchterscheinung mit vollständig bedeckter Kathodenoberfläche. Es ist dieses Stadium eine anomale Glimmentladung. Dies wird deutlich, wenn man die stationäre Strom-Spannungscharakteristik vom Lawinen-(Dunkelstrom-) bis zum Bogenstadium betrachtet, die in Abb. 7 schematisch aufgetragen ist. Die untersuchten Stromanstiege beginnen in diesem Diagramm im Punkte Z im Lawinenstadium. Da die an der Strecke liegende Spannung während der Stromentwicklung konstant gehalten wurde, steigt der Strom über den Bereich der normalen

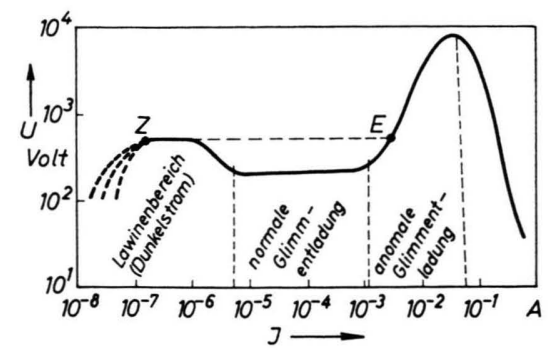

Abb. 7. Stationäre Strom-Spannungscharakteristik (halbschematisch). Der gestrichelte Verlauf von $\mathrm{Z}$ nach $\mathrm{E}$ zeigt den bei unseren Messungen durchlaufenen Bereich.
Glimmentladung hinaus und erreicht erst wieder in E, dem Bereich der anomalen Glimmentladung, die stationäre Charakteristik. Eine Entwicklung darüber hinaus ist bei der angelegten Spannung nicht möglich.

Es liegt nahe, auch im Kathodenfall der normalen Glimmentladung zwischen ebenen Elektroden eine ähnlich stabilisierende Wirkung der Raumladung zu vermuten, wie wir sie bei der anomalen Glimmentladung festgestellt haben. Wenn man einmal vom begrenzenden Randgebiet der normalen Glimmentladung absieht, ließe sich aus der stabilisierenden Wirkung der Raumladung die beobachtete nur teilweise Strombedeckung der Kathode deuten: Die Bedeckung wird sich gerade so einstellen, daß die Stromdichte die Höhe erreicht, bei der die Raumladung voll stabilisierend wirkt, also die Stationaritätsbedingung $\mu=1$ erfüllt ist.

Denkt man sich im Gegensatz hierzu eine gleichmäßige Stromverteilung über die gesamte Fläche, wäre bei gleichem Gesamtstrom die Stromdichte niedriger, und es wäre $\mu \neq 1$, so daß kein zeitlich konstanter Strom fließen könnte.

\section{Anhang: Zur Berechnung des Stromanstiegs}

Wegen der heute noch bestehenden Unsicherheiten in der Kenntnis der Elementardaten, besonders der Nachlieferung $\gamma$, schien uns eine näherungsweise Erfassung der Raumladungseinflüsse zur Deutung der Stromanstiege ausreichend zu sein.

Die benutzte Näherung besteht darin, daß die Ortsabhängigkeit der elektrischen Feldstärke $E(x, t)$ nicht jeweils aus der ebenfalls ortsabhängigen Elektronenund Ionenstromdichte $j_{-}(x, t), j_{+}(x, t)$ berechnet wurde, sondern daß ein mit $x$ linearer Verlauf angenommen wurde, der im Kathodenbereich eine Überhöhung und im Anodenbereich eine Absenkung des Feldes bedeutet. Diese bei hohem Druck benutzte Feldnäherung wird für das Anfangsstadium der Stromanstiege direkt aus der Porsson-Gleichung abgeleitet.

Diese lineare Feldnäherung

$$
E(x, t)=B(t) d\left(1-\frac{x}{d}\right)+\left(\frac{U}{d}-\frac{B(t) d}{2}\right)
$$

mit $B(t)=\left(1 / \varepsilon_{0} v_{+}\right) j(t) \quad\left(\varepsilon_{0}\right.$ Dielektrizitätskonstante $)$ ergibt sich aus der Porsson-Gleichung, wenn die Ionendichte als gleichmäßig über die Strecke verteilt angesehen und der geringe Einfluß der Elektronen vernachlässigt wird. Dies ist im Anfangsstadium bei $\mu \approx 1 \mathrm{er}$ füllt. Die additive Konstante ergibt sich aus der Forderung konstanter Spannung $U$ an der Strecke.

Die zeitliche Entwicklung ergibt sich aus der über die einzelne Generation gemittelten Bilanzgleichung der Elektronenstromdichte $j_{-}(x, t)$ an der Kathode $x=0$

$$
j_{-}(0, t)=\mu(t) \cdot j\left(0, t-T_{\mathrm{g}}\right),
$$

wenn $T_{\mathrm{g}}$ die Generationszeit bedeutet, also das Zeitintervall, nach dem sich jeder Lawinenvorgang reproduziert und

$$
\mu(t)=\gamma(t)\left(\exp \left[\int_{0}^{d} \alpha_{\mathrm{t}}(x) \mathrm{d} x\right]-1\right)
$$


die Rückwirkung angibt (siehe die Diskussion in ${ }^{1}$ ). Die Differenzengleichung für $j_{-}(0, t)$ wird gelöst durch

$$
j_{-}(0, t)=j_{-}(0,0) \exp \left[\int_{0}^{t} \ln \mu(t) \mathrm{d} t / T_{\mathrm{g}}\right],
$$

wenn man annimmt, daß sich $\mu(t)$ während einer $\mathrm{Ge}$ nerationszeit praktisch nicht ändert. Den Gesamtstrom erhält man bei $\gamma_{+}$-Nachlieferung durch Multiplikation mit der Gasverstärkung und der Fläche $F$ zu

$$
J(t)=j_{-}(0,0) F \exp \left[\int_{0}^{d} \alpha_{\mathrm{t}}(x) \mathrm{d} x\right] \cdot \exp \left[\int_{0}^{t} \ln \mu(t) \mathrm{d} t / T_{\mathrm{g}}\right]
$$

und die momentane Stromänderung zu

$$
\frac{\mathrm{d} \ln J}{\mathrm{~d} t}=\frac{\ln \mu(t)}{T_{\mathrm{g}}}+\frac{\mathrm{d}}{\mathrm{d} t} \int_{0}^{d} \alpha_{\mathrm{t}}(x) \mathrm{d} x,
$$

wobei der zweite Term nur als Korrektur bei sehr hoher Raumladung wirksam werden kann.

Um die Stromentwicklung abhängig von der Raumladung zu erfassen, berechnet man zuerst das Feld für eine bestimmte Stromdichte $j$. Mit diesem Feld werden dann die Stoßionisation $\int_{0}^{d} \alpha_{\mathrm{t}}(x) \mathrm{d} x$ und die Nachlieferung $\gamma$ berechnet. Hierbei wurden Werte $\alpha / p(E / p)$ für Wasserstoff ${ }_{d}^{5,6}$ und Sauerstoff ${ }^{6}$ entnommen, und die Berechnung $\int_{0}^{d} \alpha_{\mathrm{t}}(x) \mathrm{d} x$ durch numerische Integration durchgeführt ${ }^{7}$. Für die Nachlieferung wurden die Werte $\gamma=2,7 \cdot 10^{-4} E / p$ für $300 \leqq E / p<2000 \mathrm{~V} / \mathrm{cm} \cdot$ Torr für $\mathrm{H}_{2}$, $\gamma=2,4 \cdot 10^{-9}(E / p)^{2}$ für $500 \leqq E / p<5000 \mathrm{~V} / \mathrm{cm} \cdot$ Torr für $\mathrm{O}_{2}$

5 D. J. Rose, Phys. Rev. 104, 273 [1956].

6 H. Sснцumbohм, Z. Phys. 184, 492 [1965].

7 Diese Beschreibung setzt das Zustandekommen einer stationären Energieverteilung der Elektronen voraus. Bei exakter Beschreibung müßten die möglicherweise schon auftretenden Abweichungen hiervon berücksichtigt werden. aus ${ }^{4}$ verwendet, die mit dem gleichen Elektrodensystem (mit unbehandelter Messingkathode) bestimmt wurden. Als maßgebliche Feldstärke wurde der Wert an der Kathode angenommen. Die erforderlichen Werte der Driftgeschwindigkeit der Ionen wurden ${ }^{8}$ entnommen.

Hieraus läßt sich nun die momentane Stromänderung durch Addition der einzelnen Anteile berechnen, denn es ist

$\frac{\mathrm{d} \ln J}{\mathrm{~d} t}=\frac{1}{T_{\mathrm{g}}}\left\{\ln \frac{\gamma}{\gamma_{0}}+\ln \frac{\exp \left[\int_{0}^{d} \alpha_{\mathrm{t}}(x) \mathrm{d} x\right]-1}{\left(\exp \left[\alpha_{0} d\right]-1\right)}+\varepsilon_{0}\right\}$,

wobei $\varepsilon_{0}$ den Wert $\varepsilon_{0} \cong \ln \mu_{0}=\ln \gamma_{0}\left(e^{\alpha_{0} d}-1\right)$ bezeichnet und $\mu_{0}$ die durch die äußere Spannung gegebene Rückwirkung ist.

Zum Vergleich mit dem experimentellen Zusammenhang $\mathrm{d} \ln J / \mathrm{d} t$ abhängig vom Strom $J$ sind $\varepsilon_{0}$ und die Fläche $F$ als Parameter festzulegen, da die Rechnung $\mathrm{d} \ln J / \mathrm{d} t$ in Abhängigkeit der Stromdichte $j$ und $\varepsilon_{0}$ ergeben hat.

Lieferte die lineare Feldnäherung negative Werte der Feldstärke im Anodenbereich, wurde statt dessen Null genommen und die Normierung neu durchgeführt. Proberechnungen mit quadratischem Feldverlauf, der im Kathodenbereich stärker, im Anodenbereich schwächer $x$-abhängig ist, gaben für die momentane Stromentwicklung innerhalb der Unsicherheiten die gleichen Werte.

Herrn Professor Dr. H. Raether danke ich für die Förderung der Arbeit.

8 H. Sснцимвонм, Z. Phys. 182, 317 [1965]. 